\title{
The chemical composition and circumstellar environment of the variable star QY Sge
}

\author{
Aruna Goswami ${ }^{1,2}$, N. Kameswara Rao ${ }^{1}$, D.L. Lambert ${ }^{3}$ \\ ${ }^{1}$ Indian Institute of Astrophysics, Bangalore 560034, India \\ ${ }^{2}$ CREST, Indian Institute of Astrophysics, Hosakote 562114, India \\ ${ }^{3}$ Department of Astronomy, University of Texas, Austin, TX \\ 78712-1083, USA
}

\begin{abstract}
Chemical composition studies of the variable star QY Sge show abundance anomalies suggestive of the pattern exhibited by the warmer RV Tau variables. Abundance analysis indicates a near-solar atmosphere with highly condensable elements depleted by several factors. Evidence of broad $\mathrm{Na} \mathrm{D}$ emission features in its spectrum, a characteristic feature of R CrB stars taken at minimum, however, raises the question of its spectral classification. We address this question through a comparative analysis of its spectrum with those of $\gamma \mathrm{Cyg}, 89 \mathrm{Her}$ and $\mathrm{R} \mathrm{CrB}$ taken at minimum, in addition to abundance analysis. We also discuss a possible physical scenario of the circumstellar environment accounting for the characteristics of the distinct spectroscopic features.
\end{abstract}

\section{Introduction}

Appearance of broad $\mathrm{NaD}$ lines in emission is a characteristic feature of $\mathrm{RCB}$ stars' spectra taken at minimum. However, QY Sge, a mass-losing G0 supergiant, exhibits these lines strongly in emission in its spectrum taken at maximum (Menzies \& Whitelock 1988). This raises the question as to whether QY Sge can be classified as an RCB star with all the associated astrophysical implications. In particular, it may be necessary to explore an evolutionary scenario, different from that operative in RCB stars. Photometrically, QY Sge is known to vary with a low amplitude and a characteristic time-scale of $50 \mathrm{~d}$. It is also known from the polarimetric studies of Trammell et al. (1994) that the local continuum is polarised, but there is a lack of polarization in the broad $\mathrm{Na} \mathrm{D}$ emission lines. This implies that the emitted photons are not scattered from the surface contributing the photospheric spectrum. Insights into the location and excitation mechanisms of the broad lines can be achieved through analysis of stars' spectra which exhibit similar features. We discuss here the spectrum and abundance analysis of QY Sge with special emphasis on this aspect.

\section{Spectral characteristics}

A comparative analysis of the spectrum of QY Sge was conducted by comparing its spectrum with those of $\gamma \mathrm{Cyg}$, a normal F8 Iab star, 89 Her, a warmer 


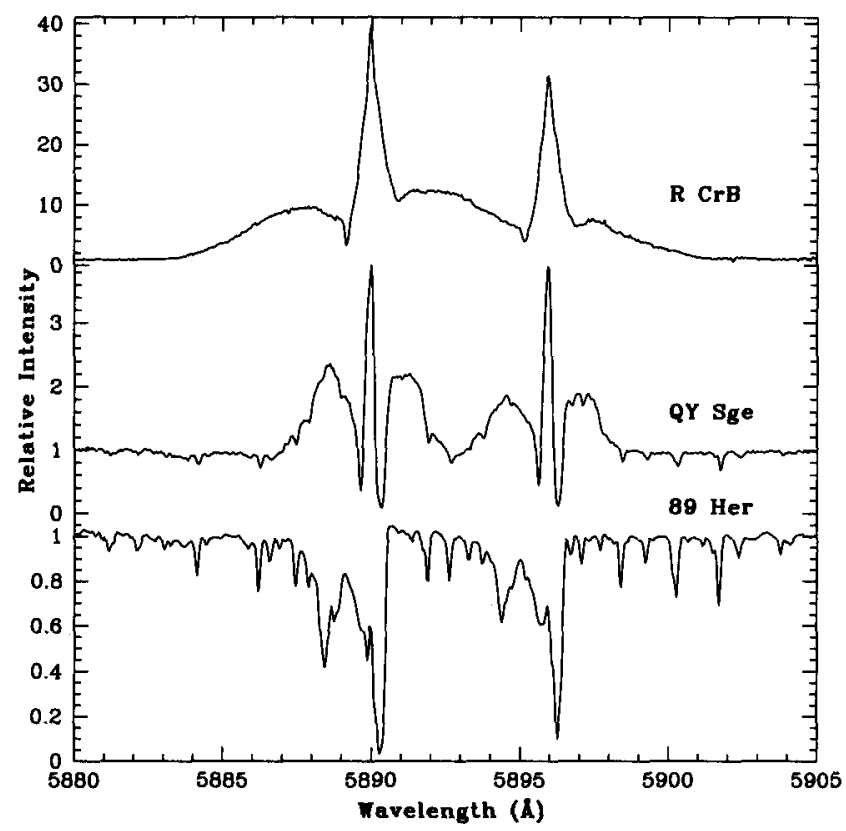

Figure 1. A comparison of the NaD features in the spectra of $89 \mathrm{Her}$, QY Sge and R CrB at minimum.

supergiant of spectral type F2 Ib, and R CrB's spectrum taken at minimum. The wavelength region spans from 3900 to $10000 \AA$ and the spectral resolving power is of $\sim 60000$. The spectra were taken with the McDonald Observatory 2.7-m Harlan J. Smith telescope with its $2 \mathrm{~d}$ coude' cross-dispersed echelle spectrograph (Tull et al. 1995) during the period 1999 to 2002. The spectrum of QY Sge is characterized by three distinct components: (i) Absorption lines representative of a late- $F$ and early-G supergiants,(ii) sharp emission lines from resonance and low-excitation transitions of abundant neutral atoms, and (iii) a few broad emission lines. The absorption line spectrum broadly resembles that of $\gamma \mathrm{Cyg}$; however, the lines in QY Sge are much broader. This broadening is plausibly attributed to the receipt of photons scattering off moving dust grains in the stellar wind. The similarities of QY Sge's spectrum with that of $89 \mathrm{Her}$, a spectroscopic binary with a low-mass companion, to the post-AGB primary is also remarkable. Both the stars have similar $\mathrm{H} \alpha$ profiles, similar sharp lowexcitation emission lines of neutral metals, and a considerable infrared excess. To list a few prominent differences, 89 Her shows a blue-shifted high-velocity absorption at $\mathrm{Na} \mathrm{D}$, whereas these features appear in QY Sge in broad emissions (Fig. 1); the low excitation emission lines seen in QY Sge's spectrum are less common than in the spectrum of $89 \mathrm{Her}$ and broader than their $89 \mathrm{Her}$ counterparts (Fig. 2, left panel). 

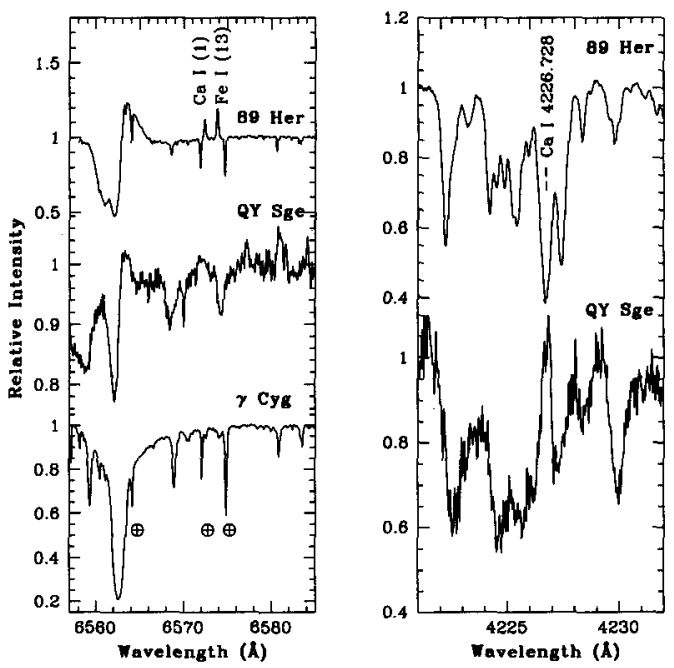

Figure 2. A comparison of the $H \alpha$ feature in $89 \mathrm{Her}, \mathrm{QY}$ Sge and $\gamma$ Cyg (left panel). The CaI feature at $4226.7 \AA$ appears in emission in QY Sge; this feature is seen in absorption in 89 Her (right panel).

The most prominent broad emission profiles $\mathrm{NaD}$ and $\mathrm{KI}$ resonance lines are similar to their counterparts in R CrB's spectrum taken at minimum. For $\mathrm{R} C r \mathrm{~B}$ the broad emission of red-wing of $\mathrm{D} 2$ overlaps the blue wing of $\mathrm{D} 1$, but $\mathrm{D} 1$ and D2 are well resolved for QY Sge. Also, among the sharp emission lines the most prominent lines are $\mathrm{NaD}$ and $\mathrm{K} I$ resonance lines. The superposition of the sharp emissions and accompanying absorptions in QY Sge otherwise creates the appearance of a single peaked broad emission split into a blue and red component of approximately equal intensity (Fig. 1).

The weak emission lines and the absorption lines from the same low-excitation levels are at a velocity of about $-14 \mathrm{~km} \mathrm{~s}^{-1}$; red-shifted by about $7 \mathrm{~km} \mathrm{~s}^{-1}$ with respect to the absorption-line velocity measured from higher excitation lines. The broad emissions of QY Sge are approximately centered on the stellar velocity. The star is a velocity variable (Rao et al. 2002).

\section{The chemical composition of QY Sge}

The atmospheric parameters were estimated using a standard model atmosphere in local thermodynamic equilibrium (LTE). The values of effective temperature $T_{\text {eff }}$, surface gravity $\log g$, microturbulence $\xi_{t}$, and the iron abundance $[\mathrm{Fe} / \mathrm{H}]$ were found to be respectively as $5850 \pm 200 \mathrm{~K}, 0.7 \pm 0.25$ in cgs units, $4.5 \pm$ $0.5 \mathrm{~km} \mathrm{~s}^{-1}$ and $-0.4 \pm 0.1$. The details are available in Rao et al. (2002). There is no evidence that QY Sge is hydrogen deficient and this ruled out QY Sge as an RCB star. $\mathrm{C}, \mathrm{N}, \mathrm{O}, \mathrm{Na}$ and $\mathrm{S}$ are overabundant and $\mathrm{Sc}, \mathrm{Ti}, \mathrm{Y}, \mathrm{Zr}$, Ce are underabundant. Heavy elements are noted to possess unusual relative 
abundances. $\mathrm{Y}, \mathrm{Zr}$ and $\mathrm{Ce}$ are underabundant by about 1 dex, but $\mathrm{Ba}$ and $\mathrm{Eu}$ show approximately solar abundances. These abundance anomalies are similar in nature to the pattern of abundances shown by a large number of warmer RV Tau variables. In particular, as in the case of warmer RV Tau variables QY Sge also shows a clear tendency for the elements of highest condensation temperature to be underabundant relative to those of lower condensation temperature. In addition, the report of QY Sge's photometric variations on a characteristic timescale of $50 \mathrm{~d}$ could also be a sign that QY Sge is a RV Tau variable.

QYSge appears to maintain a nearby reservoir of dust and have a photosphere reduced in condensable elements. The photosphere has approximately normal abundances of elements such as $\mathrm{Si}, \mathrm{Mg}$ and $\mathrm{Fe}$, the principal constituents of the circumstellar dust.

\section{Concluding remarks}

QY Sge's infrared excess indicates the presence of a thick dust cloud. A plausible scenario of the circumstellar dust is one in which the bulk of the dust forms an equatorial torus with its inner edge close to the star. With the dust optically thick along the line of sight, the contribution to the observed optical spectrum from directly transmitted photospheric light will be negligible. When the line of sight is inclined slightly to the plane of the torus, the rear of the inner edge of the torus becomes visible, but not the star. Starlight would thus reach the observer after scattering off the inner edge of the dusty rotating torus. A bipolar wind off the star probably is the site for the formation of the sharp emission lines near the star and broad emission lines farther from the star.

Although the spectral characteristics and, in particular, the appearance of broad $\mathrm{NaD}$ emission features in the spectrum of QY Sge are very similar to their counterparts in R CrB's spectrum taken at minimum, it is not a hydrogendeficient star and hence does not belong to the RCB class. The geometry of the circumstellar material, as well as the location and formation mechanisms of these broad lines, may be a common one, but their evolutionary paths are likely to be distinct. Further photometric and spectroscopic observations are desirable to investigate whether QY Sge is a pulsating star of RV Tau type and/or a spectroscopic binary.

Acknowledgments. Aruna Goswami is grateful to IAU and INSA for supporting her visit to Christchurch, New Zealand, to attend IAU Colloquium 193 on "Variable stars in the Local Group".

\section{References}

Menzies, J.W., Whitelock, P.A. 1988, MNRAS, 233, 697

Rao, N.K., Goswami, A., Lambert, D.L. 2002, MNRAS, 310, 717

Trammell, S.R., Dinerstein, H.L., Goodrich, R.W. 1994, AJ, 108, 984

Tull, R.G., MacQueen, P.J., Sneden, C., Lambert, D.L. 1995, PASP, 107, 251 


\section{Discussion}

Lloyd Evans: What are the infrared properties of this star?

Goswami: JHKL photometry by Menzies and Whitlock indicates that the star is a variable with a period of $50 \mathrm{~d}$; the infrared excess signals the presence of a thick dust cloud for which Menzies and Whitelock estimated a blackbody temperature of about $600 \mathrm{~K}$.

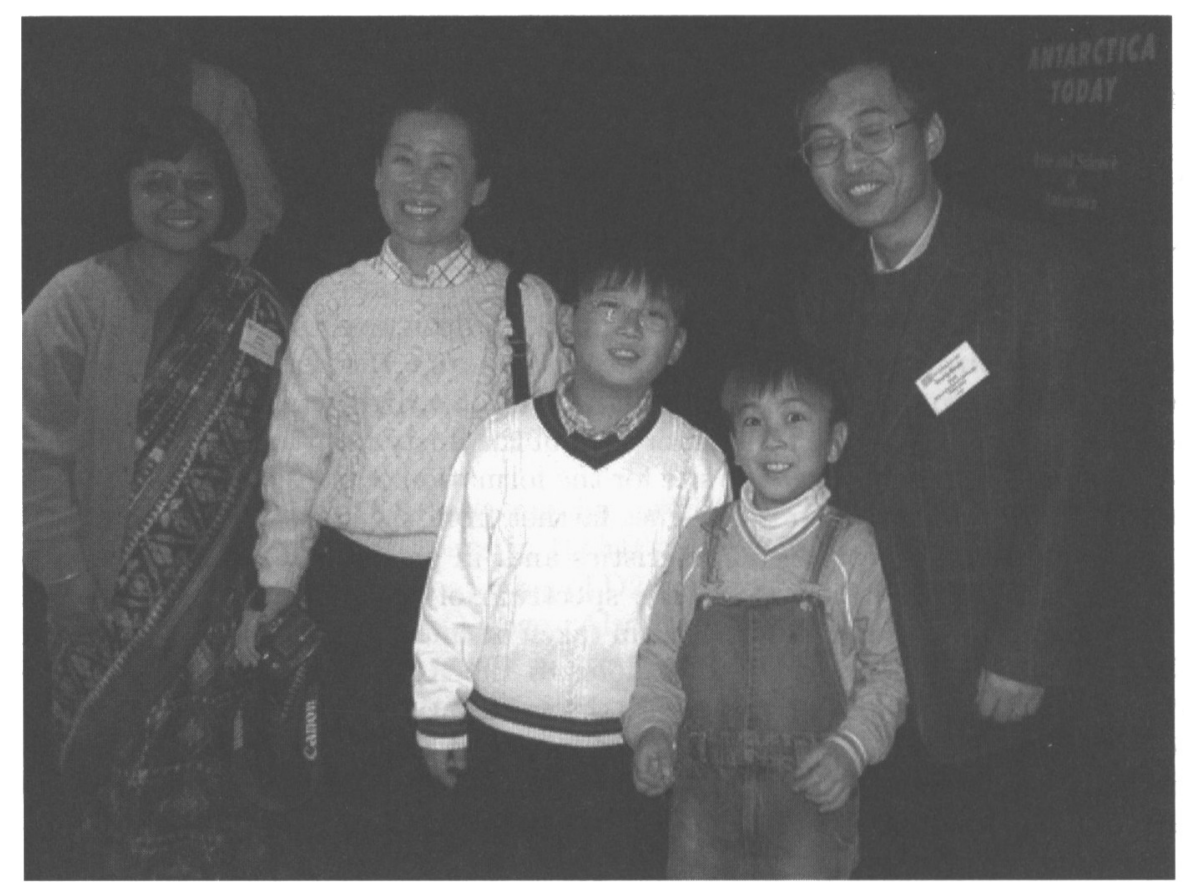

Aruna Goswami, Young-Beom Jeon and family 